\title{
Guided Bone Regeneration in the Oral Cavity: A Review
}

\author{
Rita A. Hitti and David G. Kerns ${ }^{*}$ \\ Department of Periodontics, Texas A \& M Health Science Center, Baylor College of Dentistry, Dallas, Texas, USA
}

\begin{abstract}
Guided bone regeneration (GBR) in the oral cavity is defined according to the American Academy of Periodontology as "procedures attempting to regenerate lost periodontal structures through different tissue responses... typically referring to ridge augmentation or bone regeneration procedures." GBR evolved from guided tissue regeneration (GTR) techniques directed toward regenerating tissues in osseous defects adjacent to natural teeth. One of the objectives of GBR is formation of new bone at sites deficient in bone volume. Another objective is to treat fenestrations and dehiscences at implant surfaces as well as defects associated with immediate implant placement into extraction sites. GBR has allowed for placement of restorations at a more ideal location in the oral cavity, thus improving esthetics and function. This paper is a review of current techniques of GBR for ridge preservation.
\end{abstract}

Keywords: Guided bone regeneration, ridge preservation, implants, dental implants, extractions.

\section{INTRODUCTION}

One of the initial objectives of periodontal therapy is infection management.

Understanding the putative pathogenic periodontal microfloralanguage has altered therapeutic approaches from one of elimination of microbes to one of controlling pathogenic microorganisms and the immuno-inflammatory response. Using treatments such as scaling and root planning, maintenance therapy, and antimicrobial therapy, the main goal is to control the pathogenic microflora to prevent further periodontal destruction.

Despite successful disease control, anatomic changes resulting from past disease activity often occur and need to be corrected. Therapeutic approaches include procedures such as flap debridement/flap curettage, resective procedures, and periodontal regenerative therapy. Of these therapies, periodontal regeneration is the ideal goal [1].

Melcher [2] contributed to the current understanding of periodontal healing. It is based on a hypothesis that the cell type that repopulates the exposed root surface at the periodontal repair site will define the nature of the attachment or repair that takes place. If mesenchymal cells from the PDL or perivascular region of the bone proliferate and colonize the root surface, regeneration occurs. If epithelial cells proliferate along the root surface, a long junctional epithelium will result. If gingival connective tissue populates the root surface, a connective tissue attachment will form and root resorption may occur. If bone cells migrate and adhere to the root surface, root resorption and ankylosis occur. Root resorption is much more common in animal models than it is in humans. Animal models have confirmed the importance of periodontal ligament (PDL) cells as progenitor cells for periodontal regeneration $[3,4]$.

*Address correspondence to this author at the Department of Periodontics, TAMHSC - Baylor College of Dentistry, 3302 Gaston Avenue, Room 141, Dallas, Texas 75246, USA; Tel: 214-828-8140; Fax: 214-874-4532;

E-mail: DKerns@bcd.tamhsc.edu
Evaluation of cell proliferation kinetics revealed that both the PDL and perivascular cells from the bone proliferate and migrate into the osseous defect to form the early healing tissue [5]. Melcher [6] amended his original hypothesis to include the contribution of the perivascular cells of the bone in periodontal regeneration concluding that cells from both the PDL and alveolar bone are important in formation of new bone, cementum, and functionally oriented PDL (regeneration).

The mechanism of new attachment formation was described by a series of studies by Nyman, Karring, Lindhe and others [7-9], and based on this, guided tissue regeneration (GTR) was designated as a periodontal tissue regenerative procedure.

Nyman et al. [9] treated eleven periodontally involved teeth in ten (10) patients and performed periodontal therapy without osseous surgery. A teflon membrane $\left(\right.$ Gore-Tex ${ }^{\mathbb{B}}$ ) was placed on the coronal one- third of the root. The mucoperiosteal flaps were secured with sutures over the membrane. Three months after healing, four teeth were extracted with their associated periodontal tissues and examined histologically. New attachment was evident in five sections. The seven non-extracted teeth were evaluated for attachment level at a second surgical procedure three months later at which time the membrane was removed. Three months later, using clinical parameters, probing attachment levels were re-evaluated. In some teeth, new attachment formed, whereas in others, only a few millimeters of new cementum formed.

These results demonstrated the basis of the biologic principle of GTR for the treatment of periodontal disease in humans. Currently, the periodontal literature is replete with basic research and clinical investigations concerning GTR for the treatment of localized bone defects in natural dentition and in conjunction with endosseous implants.

The osteopromotion principle describes the technique of physically placing a barrier over sites of osseous deformities where insufficient vertical, buccal and/or lingual bone 
volume is present. This segregates the site from the surrounding soft tissues, which are known to interfere with osteogenesis or the formation of new bone [10]. The use of this term is germane to the terminology that describes bone forming mechanisms, such as osteoinduction, osteoconduction, osteogenesis, and osseointegration. As discussed previously, the use of the barrier membrane enhances complete osteogenesis by preventing the rapid ingrowth of fibroblasts into a bony defect and promoting the migration of osteogenic cells from the adjacent bony edges or bone marrow into the defect in an unimpeded fashion (Fig. 1). Leaving the barrier membrane in place for an extended period of time and sealing off the bony defect permits uninterrupted osteogenesis to occur and allows maturation of the newly formed bone.

The rationale of GTR is to impede apical migration of the epithelium by placing a barrier membrane (epithelial exclusion), which can allow the repopulation of PDL cells onto the dental root surface.

\section{Guided Bone Regeneration}

Dahlin and colleagues [11-13] spearheaded early research on guided bone regeneration (GBR) in an attempt to solve the confounding problem of reconstructing large, osseous defects in the jaws and for the treatment of the atrophic maxilla or mandible. It is known that to accomplish the repair of a bone defect, the rate of osteogenesis extending inward from the adjacent bone ends must exceed the rate of fibrogenesis growing in from the surrounding muscle or connective tissue [14].

In 1988, Dahlin et al. [12] published the results of animal experimentation on the healing of bone defects. Bilaterally, a through-and-through defect was surgically created in the ramus in 30 Sprague-Dawley rats. On one side of the jaw, the defect was covered with a porous polytetrafluoroethylene (PTFE) membrane (Gore-Tex ${ }^{\circledR}$ ). The other side served as the control, without a membrane covering. After 3, 6, and 9 weeks of healing, the specimens were evaluated macroscopically and histologically by light microscope. Statistical analysis of the healed sites demonstrated a highly significant increase in bone regeneration on the membrane side as compared to the control. Therefore, it was demonstrated that soft tissue in-growth into a bony defect could be prevented and consequently could enhance unimpeded bone healing greatly.

Further experimentation was performed in animals which demonstrated the generation of bone around titanium implants.

As an alternative to a two-stage, bone grafting procedure followed by implant placement, Dahlin et al. [13] evaluated the principle of GTR to generate bone at the exposed parts of titanium implants. Thirty "commercially pure" $10-\mathrm{mm}$ titanium implants were placed in the tibia of 15 adult rabbits, each with three to four exposed threads per implant. A PTFE membrane was placed over the test fixtures, covering the threads and 5 to $8 \mathrm{~mm}$ of the adjacent bone. The muscle and periosteum were replaced, adapted, and sutured. The control fixtures were not covered with a membrane. After healing periods of 6,9 , and 15 weeks, the specimens were removed en bloc and evaluated grossly and histologically. The results showed that all exposed threads of the titanium implants were covered with newly formed bone at a uniform thickness, even as early as 6 weeks. New bone formation also was seen in the control areas, although to a much lesser extent than the test areas. It was shown that by placing an inert membrane with an appropriate pore size, which hindered the penetration of undesirable cells, a space was created that permitted the entrance of osteogenic and angiogenic cells from the adjacent bone marrow to populate the area and proliferate. It also was recognized that the amount of new bone formed was contingent upon the amount of space created by the membrane.

Further studies by Dahlin et al. [11] continued to provide support for the principle of guided bone regeneration in the regeneration of bone for clinical application.

Osseous defects surgically created in the maxilla and mandible of monkeys, which were covered by a barrier membrane, evidenced complete regeneration of bone.

The principle of selective cell repopulation has been useful in enhancing site development for implant placement [1]. Whereas GTR requires the regeneration of bone, PDL and cementum to form a new periodontal apparatus, the requirements for implant site development are less complicated in that only bone formation needs to be enhanced. By using a barrier membrane at an extraction site

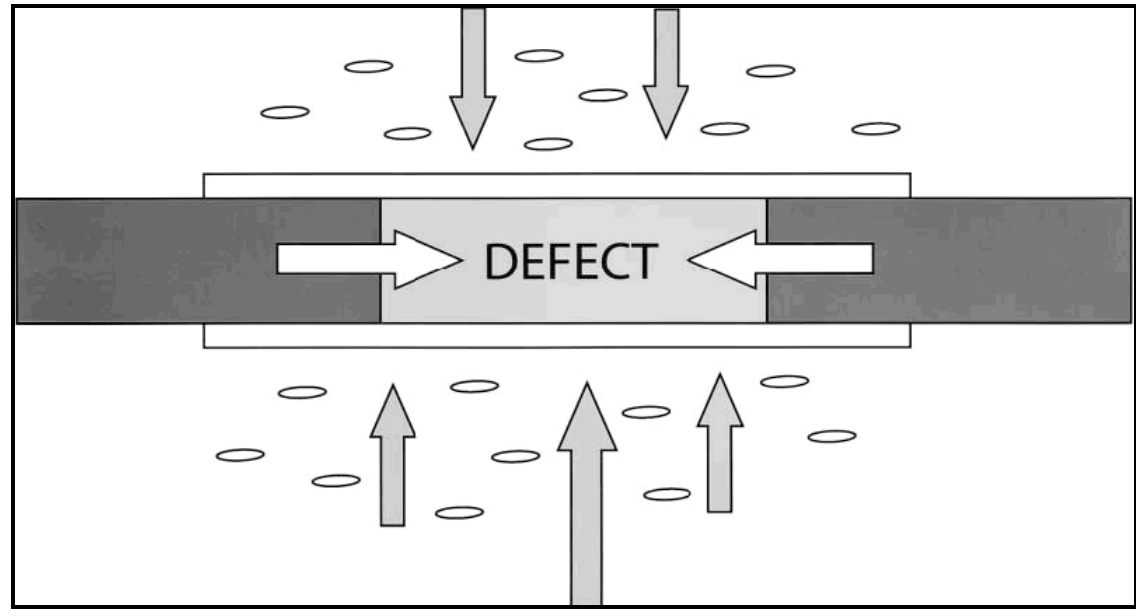

Fig. (1). Osteopromotion principle. 
or deficient alveolar ridge, bone can be regenerated. At the time of tooth extraction, the socket can be augmented with a graft material and sealed with a barrier membrane. In some cases, a membrane may be used without graft material in the socket. $\mathrm{T}$ his procedure is termed ridge preservation 1 . Similarly, an alveolar ridge with a volumetric deficiency can be improved with the use of graft material and a barrier. This procedure is also termed guided bone regeneration (GBR) and is a commonly used technique for osseous ridge augmentation [1].

Sufficient alveolar bone volume and favorable architecture of the alveolar ridge are essential to obtain ideal functional and esthetic prosthetic reconstruction following implant therapy [15]. Knowledge about the healing process at extraction sites, including contour changes caused by bone resorption and remodeling, is essential.

Loss of alveolar bone may occur prior to tooth extraction because of periodontal disease, periapical pathology, or trauma to teeth and bone. Damage of the bone tissues during tooth extraction procedures may also result in bone loss. Finally, alveolar bone atrophy after tooth extraction is a well-known phenomenon.

Schropp15 demonstrated that major changes of an extraction site take place during the 12 months following tooth extraction. The width of the alveolar ridge was reduced by $50 \%$ during the observation period. This loss, corresponding to 5 to $7 \mathrm{~mm}$, is in agreement with earlier studies. The finding that approximately two thirds of this reduction occurred within the first 3 months after tooth extraction also corresponds to earlier findings. When analyzing the extraction sites separately according to region and jaw, there was no major diversity between the sites.

A 6-month randomized, controlled, blinded clinical study [16] was conducted to determine whether ridge preservation would prevent post-extraction resorptive changes as assessed by clinical and histologic parameters. Twenty-four patients aged 28 to 76 years, requiring a non-molar extraction and delayed implant placement were randomly selected to receive either extraction alone (EXT) or ridge preservation (RP) using tetracycline hydrated freeze-dried bone allograft (FDBA) and a collagen membrane. Following extraction, horizontal and vertical ridge dimensions were determined using a modified digital caliper and an acrylic stent respectively. Prior to implant placement, a $2.7 \times 6.0 \mathrm{~mm}$ trephine core was obtained for histologic analysis.

The width of the RP group decreased from 9.2 to 8.0 $\mathrm{mm}$, while the width of the EXT group decreased from 9.1 to $6.4 \mathrm{~mm}$, a difference of $1.6 \mathrm{~mm}$. Both the EXT and RP groups lost ridge width, although an improved result was obtained in the RP group.

Most of the resorption occurred from the buccal and maxillary sites lost more width than mandibular sites. The vertical change for the RP group was a gain of $1.3 \mathrm{~mm}$ versus a loss of $0.9 \mathrm{~mm}$ for the EXT group, a height difference of 2.2 $\mathrm{mm}$. Histologic analysis revealed more bone in the RP group: about $65 \%$ versus $54 \%$ in the EXT group. The RP group included both vital bone (28\%) and non-vital (37\%) FDBA fragments. They concluded that ridge preservation using FDBA and a collagen membrane improved ridge height and width dimensions when compared to extraction alone.
Rationales for augmenting bone formation primarily relate to improvement in function and esthetics of restorative dentistry [17]. GBR may also be used as an adjunctive procedure to enhance conventional dental prosthetics such as a fixed partial denture. An improvement of deficient contours beneath a pontic of a fixed partial denture will facilitate more natural emergence profiles as well as prevent altered phonetics due to space between the restoration and the gingival tissues.

Additional indications for bone augmentation include increasing the volume of bone in order to facilitate placement of endosseous dental implants. GBR procedures can be accomplished prior to or in some cases in conjunction with dental implant placement. In other cases, the purpose is to increase bone volume where deficiency would compromise the function or esthetics of the dental implant restoration [17].

\section{PRINCIPLES OF GUIDED BONE REGENERATION}

To achieve better clinical outcomes, the GBR barrier should possess the following properties [18]:

Cell exclusion: In GBR, the barrier membrane is used to prevent gingival fibroblasts and/or epithelial cells from gaining access to the wound site and forming fibrous connective tissue.

Tenting: The membrane is carefully fitted and applied in such a manner that a space is created beneath the membrane, completely isolating the defect to be regenerated from the overlying soft tissue. It is important that the membrane be trimmed so that it extends 2 to $3 \mathrm{~mm}$ beyond the margins of the defect in all directions. The corners of the membrane should be also rounded to prevent inadvertent flap perforation.

Scaffolding: This tented space initially becomes occupied by a fibrin clot, which serves as a scaffold for the in-growth of progenitor cells. In GBR, the cells will come from adjacent bone or bone marrow.

Stabilization: The membrane must also protect the clot from being disturbed by movement of the overlying flap during healing. It is therefore often, but not always, fixed into position with sutures, mini bone screws, or bone tacks. Sometimes, the edges of the membrane are simply tucked beneath the margins of the flaps at the time of closure, providing stabilization.

Framework: where necessary, as in non-space maintaining defects such as dehiscences or fenestrations, the membrane must be supported to prevent collapse.

Bone-replacement grafts are often used for this purpose. They serve as a sort of internal framework to provide a measure of support to the graft. Stiffer membranes such as titanium-reinforced membranes have also been used for this purpose.

\section{FACTORS AFFECTING SUCCESSFUL REGENERATION}

A number of factors have been implicated or shown to adversely influence periodontal regeneration therapy 19. These include: 
Bacterial contamination: It is well established that plaque control is a critical determinant of the success or failure of various outcomes of periodontal therapy [19].

Tissue-implanted materials, such as GTR membranes, encourage bacterial contamination of the local site [20]. Mombelli et al. [21] evaluated the microbial contamination of expanded polytetrafluoroethylene (e-PTFE) membranes 6 weeks after surgical placement in ten patients. Patients received diligent professional monitoring during the postoperative healing phase, including professional cleaning at least every week and home rinsing with chlorhexidine $(0.1 \%)$. Surgical results were described as clinically successful, yet gram-negative anaerobic rods, commonly associated with adverse periodontal conditions, were found in all samples.

Porphyromonas gingivalis was found at high levels in one patient, and Prevotella intermedia was found in six of the nine patients. The authors concluded that GTR membranes appeared to harbor periodontal pathogens on a frequent basis. Several investigators have found relatively high levels of bacterial contamination of e-PTFE membranes $[22,23]$. Others [24] have reported that guided tissue regeneration sites during the active healing phase were more likely to be colonized by periodontal bacteria than sites treated without membranes.

In clinical sites with submerged barrier membranes, periodontal pathogens were not present by various detection techniques, whereas high proportions of $P$. gingivalis, Actinobacillus actinomycetemcomitans (Aa) and Peptostreptococcus micros were found in exposed membranes with minimal bone regeneration [25].

The type of membrane used for regeneration in human subjects, including collagen, e-PTFE and polylactic acid, does not seem to influence the colonization by various periodontal bacterial species [26].

Experimental studies of GTR in monkeys have explored the influence of membrane exposure to bacterial plaque on the healing of the lesions. In one study [27], experimental periodontal lesions were created and the GTR membranes were either completely covered by soft tissue or left exposed by $2 \mathrm{~mm}$. After 6 months of healing, histological evaluation showed that the covered GTR membranes had new connective tissue and bone corresponding to $67 \%$ to $100 \%$ of the initial depth of the defect, whereas the healing under exposed membranes ranged from $30 \%$ to $59 \%$ of the defect depth. Although this study suggests a major role for the bacteria in less complete regeneration, the exposed membrane also introduces additional complications relative to healing, such as differences in revascularization.

The clinical effects of plaque control have been well described in longitudinal studies of GTR procedures. In one 4-year study [28] of 23 patients, GTR procedures resulted in a mean gain of $4.1 \mathrm{~mm}$ of clinical attachment level after 1 year of strict plaque control. This clinical outcome was stable for an additional 3 years in 15 patients who adhered to a regular recall program every 3 months. In the other 8 patients who received sporadic maintenance care, a mean of $2.8 \mathrm{~mm}$ of the 1-year gain was lost in the next 3 years. In addition, $P$. gingivalis and $P$. intermedia were detected more frequently in the sporadic care group. Machtei et al. [29] surgically reentered mandibular class II furcations that had been treated with guided tissue regeneration therapy and evaluated the success factors. They determined that the optimal gain in attachment level and the amount of new bone were observed in sites that met the following criteria: 1) deeper sites, 2) good oral hygiene, 3) minimal inflammation, 4) no detectable $\mathrm{Aa}$ and 5) the presence, by microscopy, of connective tissue cells on the inner surface of the membrane. In a similar 1-year longitudinal study [30], 47\% of the variability in clinical attachment level could be explained by defect characteristics, early membrane exposure and the presence of plaque in the area. The presence of plaque in the local area was associated with significantly less clinical attachment level gain and less bone fill. Hugoson et al. [31] have noted that high plaque levels were present in many of the class II furcations that were treated with guided tissue regeneration procedures, which did not respond favorably.

Smoking: In a retrospective analysis of a longitudinal study of GTR procedures in class II furcations, Rosenberg et al. [32] reported a $42 \%$ failure rate after at least 4 years. Of those failures, however, $80 \%$ were in patients who smoked at least 10 cigarettes per day for 5 years.

As part of an extensive clinical study to define success criteria and determinants for regeneration, Tonetti et al. [33] determined the influence of smoking on regeneration outcomes. At 1 year after GTR surgery, smokers $( \pm 10$ cigarettes per day) had a significantly less favorable gain in probing attachment level than did nonsmokers.

The probing attachment level gain in non-smokers was $5.2 \pm 1.9 \mathrm{~mm}$ compared with $2.1 \pm 1.2 \mathrm{~mm}$ gain in smokers. This effect remained significant after adjustment for different plaque levels and baseline differences in defect anatomy.

Approximately $62 \%$ of the variance in the probing attachment level outcome could be explained by the baseline depth of the infrabony defect, smoking status and oral hygiene. Smoking produced a 4.3 times increased risk of an unfavorable response. In patients who were nonsmokers and had good oral hygiene, only $8.7 \%$ of the sites had an unfavorable outcome after one year. In patients who were smokers, had poor oral hygiene, or both risk factors, $43.8 \%$ to $62.5 \%$ of the sites had an unfavorable response.

In a 5-year follow-up in a controlled study [34], GTR therapy was compared to root planing alone. In most cases both the GTR site and the control site in each patient responded concordantly. Patients in which both sites remained stable were characterized by good oral hygiene, compliance with recall, and non-smoking status.

Those in which both sites showed deterioration tended to be smokers with oral hygiene that deteriorated during the follow-up period.

Diabetes: Schwartz-Arad et al. [35] determined the presence of diabetes to be a risk factor for failure in regenerative procedures, citing lower success rates of block grafts in patients known to have diabetes. Kornman [19] concluded that although no direct data are available, diabetics with less than optimal glucose control should theoretically be at increased risk for failure with regenerative procedures. One component of the increased risk may be a 
delayed wound healing response that is most likely the result of poor control of glucose metabolism on the inflammatory process. Improved metabolic control is currently the only practical approach to managing this risk factor. There are currently no data to quantify the influence of diabetes on the success of regeneration.

Defect morphology and tooth anatomy: The number of associated bony walls and overall defect depth has long been related to success of regenerative therapy. In a retrospective analysis of 26 proximal defects treated with flap surgery and e-PTFE barrier membranes, Selvig et al. [22] concluded that the extent of crestal involvement, circumference, number of tooth walls involved, and wall form in the fundus of the defect did not influence the healing response. Attachment gain and bone fill were positively correlated with the depth of the 3-walled intraosseous component of the defect. A series of studies focused on factors affecting healing of intraosseous defects treated by GTR [36, 37], also identified increased total depth of the intraosseous component of the defect as well as decreased radiographic width of the defect angle as important positive correlates of regeneration. It was suggested that the decreased amount of regeneration associated with an increased radiographic defect angle between the root surface and defect wall may reflect space loss and clot disturbance caused by postoperative collapse of the membrane, the greater distances required for cellular repopulation of the wound or an enhanced susceptibility to oral environmental factors leading to incomplete bone fill. These latter oral environmental factors, including mechanical trauma and infection, are also proposed as primary reasons for incomplete fill of the most superficial portion of the defect.

Enamel projections, bifurcation ridges, lingual grooves, irregularities in root morphology, and other factors related to tooth anatomy have been implicated in the causation of periodontal disease, primarily because they favor plaque accumulation [38].

It is reasonable to expect that such factors may also adversely affect the success of regenerative procedures [39] since the presence of plaque and related periodontal inflammation and the inability to maintain high levels of oral hygiene endanger a positive outcome of GTR.

Membrane Exposure: The most frequent postoperative complication of GTR is membrane exposure. Murphy [40] reported membrane exposure in $87 \%$ of GTR-treated sites in 62 patients (102 sites). The average time of membrane exposure was 16.2 days after surgery, with most membranes exposed within 2 weeks. The membrane exposure rate was high because Murphy used GTR for interdental osseous defects. With membrane exposure, thorough plaque control of the exposed area is essential to avoid infection. One cause of early membrane exposure is necrosis of a thin flap covering the membrane. In GTR, blood supply to the flaps depends on flap thickness because blood supply from the bone to the flap is impeded by the membrane.

The significance of early membrane exposure on the regenerative outcome in GTR and GBR procedures is somewhat controversial. Several clinical trials have shown better response when the membranes remained submerged (S) compared to those that have become exposed (E) during healing. Other studies have failed to show any such difference. In a meta-analysis conducted by Machtei et al. [41], the existing data were combined in order to provide meaningful information based on a large database.

Studies of GTR in Class II furcation and intrabony defects (IBD), together with GBR around dental implants, where the membrane became exposed during the postoperative period, were combined to form 3 separate databases. Five studies with a total of 101 sites were included in the furcation database; 43 of these sites became exposed. Mean horizontal attachment level (AL) gain for the $\mathrm{S}$ sites $(3.72+/-0.15 \mathrm{~mm})$ was slightly greater than that of the E sites $(3.06+/-0.15 \mathrm{~mm})$. For the intrabony group, there were 309 sites in 5 studies: of these, 142 sites became exposed. Mean gain in vertical AL was $4.22+/-0.15 \mathrm{~mm}$ and $4.69+/-0.13 \mathrm{~mm}$ for the $\mathrm{E}$ and $\mathrm{S}$ group, respectively. The GBR group included 60 sites in 2 studies: new bone formation in the $24 \mathrm{~S}$ sites $(3.01+/-0.38 \mathrm{~mm})$ was 6 -fold greater compared with the $36 \mathrm{E}$ sites $(0.56+/-0.45 \mathrm{~mm})$. These differences were also statistically significant. They reported an overall incidence of membrane exposure of $60 \%$ in GBR procedures. Membrane exposure during healing had a major negative effect on GBR around dental implants but only a minimal effect on GTR around natural teeth.

Early membrane exposure is a common problem in GBR during fixture placement, but it does not mean treatment failure. Simion et al. [42] examined membrane exposure in GBR and found that $99.6 \%$ bone regeneration was obtained in osseous defects around fixtures where membrane exposure did not occur for 6-8 months. They reported $48.6 \%$ bone regeneration where membrane exposure occurred earlier.

Membrane exposure reduces bone fill remarkably. Becker et al. [43] and Simion et al. [44] reported significantly less bone regeneration around implants placed into immediate implant sites when membranes became exposed compared to non-exposed membrane sites $(41.6 \%$ versus $96.6 \%$ ).

Complete primary closure is essential to prevent early exposure; however, some reports have shown that early membrane exposure may not affect the results of GBR depending on other conditions. Mellonig and Triplett [45] reported that early membrane removal did not affect results despite early membrane removal in 53\% of sites due to exposure during healing. However, frequent patient followup and thorough management of the exposed area is essential in early membrane exposure cases. Shanaman [46] reported that membrane exposure was not significant if postoperative oral hygiene management was adequate. Thus membrane exposure due to postoperative soft tissue dehiscence is disadvantageous, but thorough postoperative oral hygiene helps counter the problem because it reduces the likelihood of infection.

Gingival thickness: Anderegg et al. [47] reported on the relation between the thickness of the flap covering the membrane and gingival recession occurring after surgery. They measured the thickness of flaps $5 \mathrm{~mm}$ apical to the gingival margin during surgery and classified patients into two groups based on flap thickness. Six months after surgery, they found an average $2.1 \mathrm{~mm}$ gingival recession in 
the group with less than $1 \mathrm{~mm}$ flap thickness, and an average of $0.6 \mathrm{~mm}$ gingival recession in the group of flaps of greater thickness. The thickness of the flap covering the membrane is an essential consideration.

To maintain blood supply to the flaps, to prevent flap necrosis, and to achieve favorable results, more than $1.5 \mathrm{~mm}$ gingival thickness is a prerequisite in GTR. If GTR is performed in deep osseous defects with thin gingival in the maxillary anterior region, recession of the interdental papilla or gingival will occur. Therefore, for esthetic reasons, other procedures, such as flap curettage or bone grafts, should be considered [48].

Space maintenance: Space maintenance is considered a desirable property in a barrier device, with a direct correlation between bone volume regenerate and potential volume beneath a membrane. Jovanovic et al. [49] reported bone regeneration within localized alveolar ridge defects using a flexible, titanium-reinforced e-PTFE membrane that can be shaped to conform to the desired ridge morphology with the purpose of preventing membrane collapse within the ridge defect. In addition to membrane rigidity, other means of providing space maintenance include the use of tenting screws, osseous particulate grafts, cortico-cancellous osseous block grafts, dental implants, and use of binding agents in combination with osseous graft materials. Currently, there are no available collagen-based membranes with enough structural rigidity to maintain shape over the defect alone. In cases where these membranes are used to treat ridge defects, graft material is placed into the ridge deformity to support the overlying collagen membrane, thus facilitating space maintenance.

\section{BARRIER MEMBRANES}

There are five criteria considered important in the design of barrier membranes used for GTR [50,51]. These include biocompatibility, cell-occlusiveness, space making, tissue integration and clinical manageability. Various types of materials have been developed, which can be grouped together as either non-resorbable or resorbable membranes.

\section{NON-RESORBABLE MEMBRANES}

The first membranes used experimentally by Nyman's group in their initial work were constructed from Millipore ${ }^{\circledR}$ (cellulose acetate) filters. As this technique became more prevalent, the first commercial membrane was produced from Teflon ${ }^{\circledR}$ (e-PTFE). This membrane consisted of 2 parts: a collar portion, having open pores to allow in-growth of connective tissue and to prevent epithelial migration; and an occlusive portion, preventing the flap tissues from coming into contact with the root surface [52]. Because the space defined and protected by the membrane determined the volume of tissue that could be regenerated, the material was redesigned with a stiff central portion to treat osseous defects [51,52] and reinforced with titanium for both osseous and periodontal defects [51]. Successful use of non-resorbable membranes in GTR therapy led to application of these membranes in GBR procedures.

\section{e-PTFE Membrane in GBR-Ridge Augmentation}

Buser [53] was one of the first to report successful ridge augmentation with GBR in humans using an e-PTFE membrane and tenting pins. He reported on twelve patients who received alveolar ridge augmentation for the purpose of dental implant placement. Surgical protocol included mucoperiosteal flap reflection followed by perforations of the cortical plate within the defect using a round bur to increase blood supply to the graft. Placement of titanium mini-screws within the defect helped to provide support to the overlying e-PTFE membrane. Following a healing period of six to ten months, the authors demonstrated an increase in bone volume adequate to allow placement of dental implants in nine of the twelve sites. The gain in new bone formation ranged from 1.5 to $5.5 \mathrm{~mm}$. The authors concluded that the biologic principle of osteopromotion by exclusion is highly predictable for ridge enlargement or defect regeneration under the prerequisite of complication-free healing. Schenk [54] studied the pattern of bone regeneration in membraneprotected, critical size defects in the mandible of four adult male foxhounds. Extraction of three mandibular premolars bilaterally created a $50 \mathrm{~mm}$ edentulous space. Four months after the extractions, membrane surgery was performed. Two through-and-through rectangular defects were created bilaterally that measured approximately $8 \mathrm{~mm}$ vertically, 12 $\mathrm{mm}$ mesiodistally, and $10 \mathrm{~mm}$ buccolingually at the most inferior aspect of the defect. Each dog received (1) one standard, e-PTFE membrane, (2) two prototype reinforced ePTFE membranes (r-GTAM) that had been preformed into an arch shape, and (3) one non-membrane control site. The membranes were sized to cover the defect and fit beyond the bony margin by 2 to $3 \mathrm{~mm}$. The membranes also were secured to the alveolus with fixation screws. Primary wound closure was carried out. Healing was permitted for 2 months in two dogs and 4 months in the remaining two dogs. Each of the four control sites demonstrated incomplete bone regeneration restricted to the bony margins of the defect. Copious scar tissue formation also was present within the defect. A deep indentation persisted along the alveolar crest that was partially filled by the collapsed mucosa, a finding not observed in the membrane-covered defects. The microscopic pattern of bone formation occurred, as demonstrated in the 2-month and 4-month healing specimens, in three general categories or phases. The most immature bone was woven bone, a random orientation of collagen fibrils along the margins of the surgically created defect. Next, parallelfibered bone was deposited on the surface of the primary spongiosa as reinforcement.

Finally, lamellar bone was seen, which represented the most mature phase of bone regeneration. First, this study confirms conclusions of previous experiments that demonstrated bone regeneration in membrane-protected defects. Second, the environment created by the membrane permitted the regeneration of bone by physically supporting the overlying soft tissue and preventing collapse of the soft tissue into the defect. It also protected the blood clot from surrounding soft tissue invasion while maintaining a space into which osteogenic cells could migrate and possibly enclave local growth factors and bone-promoting substances. Third, bone regeneration occurred, as mentioned earlier, in three stages. Fourth, complete bone regeneration was not seen at 4 months which provoked questions regarding the required healing time for the regenerate and the physical properties of the membrane itself, particularly of the resorbable type. Finally, the reinforced membranes 
maintained their original structure throughout the study as compared to the e-PTFE membrane, which showed minor deformation from the overlying soft tissue.

Fifteen non-submerged implants were placed bilaterally in regenerated bone created in membrane- protected defects in the mandible of five adult foxhounds [55]. A 6-month healing period occurred between the surgical creation of the defects and the placement of the implants. 8 implants were restored and functionally loaded for 6 months, leaving 7 implants unrestored. At the termination of the study, 9 months after implant placement, the histologic analysis demonstrated direct bone to implant contact for all 15 implants. It may be concluded that bone regenerated under a barrier membrane responds to implant placement similarly to native, non-regenerated bone by stimulating bone maturation and remodeling. The newly formed bone also is capable of sustaining functional loading. Interestingly, the regenerated bone that served as a control (no implant placed) demonstrated bone atrophy beneath the membrane.

Becker et al. [43] conducted a prospective multicenter clinical study that determined the predictability for implants placed into immediate extraction sockets and augmented with e-PTFE barrier membranes. A total of 49 implants were placed with e-PTFE membranes. Three implants were lost at abutment connection surgery.

Patients were followed up to 1 year after implant loading. The average bone formation for membrane-retained sites was $4.8 \mathrm{~mm}$, whereas the average bone formation for sites in which the membranes were prematurely removed (20) was 4 $\mathrm{mm}$. At stage 2 surgery, an average of 0.6 threads were exposed for the membrane retained sites and 2.6 threads for the early removal sites. Forty-five pairs of nonstandard radiographs were evaluated for bone loss after implant loading ( 7.5 months). The average mesiodistal bone loss averaged $0.72 \mathrm{~mm}$. The results of this study demonstrated that by securing an e-PTFE membrane over an endosseous implant placed into an immediate extraction socket, substantial amounts of bone formation occurred adjacent to the implant. Sites at which the membranes were retained until stage 2 surgery also had the greatest amounts of bone formation.

Ridge augmentation has been performed using other materials in combination with the e-PTFE membrane, such as resorbable tenting pins or composite grafting.

Fiorellini et al. [56] evaluated the percentage of bone-toimplant contact following guided bone regeneration using ePTFE membranes and various bone fillers in a beagle dog model. Three months after bilateral extraction of the mandibular premolars and first molars, rectangular, distalextension defects that included the entire width of the ridge buccolingually were surgically created in the alveolar processes. All defects were covered with an e-PTFE membrane, and several bone fillers were placed, in a randomized fashion, under the membrane: autogenous bone, demineralized freeze-dried bone, anorganic bovine bone, tricalcium phosphate granules, and collagen sponge. One site in each animal was treated with e-PTFE barrier membrane alone as control. Following an 8-month healing period, nonsubmerged titanium implants (36 total) were placed in regenerated bone following membrane removal. Three months later, the animals were sacrificed and sections were evaluated histometrically for bone-to-implant contact. All sites demonstrated high percentages $(50 \%$ to $65 \%)$ of boneto-implant contact, with no significant differences across the various treatment groups. In addition, all tested bone fillers formed a complex that supported and maintained the osseointegrated implants in a healthy state, with no apparent signs of peri-implantitis. Using a staged approach, this study provided evidence that implants placed in entirely regenerated bone can achieve and maintain osseointegration, regardless of the type of bone fillers used.

One study [57] compared, in a human model, the ability of (1) e-PTFE membranes plus bone-chip autografts, (2) ePTFE membranes plus demineralized freeze-dried bone, (3) e-PTFE membranes plus a new form of demineralized allograft bone tissue, and (4) e-PTFE membranes alone to enhance bone regeneration around dental implants placed into recent extraction sockets. The histologic results demonstrated that, in humans, guided tissue regeneration techniques are capable of producing new bone osseointegrated with titanium dental implants. Among the graft materials, autogenous bone provided the most dense and the greatest amount of bone formation, but use of demineralized freeze-dried bone and a new form of demineralized allogenic bone matrix also improved bone regeneration compared to membranes alone after 6 months of healing. Autografts are the best material for space making, but the amount of graft material that can be harvested from the oral cavity is limited. For this reason, Nevins and Mellonig recommended the use of DFDBA [58].

The outcomes of these studies demonstrate the predictability of ridge augmentation using non-resorbable membranes either alone or in combination with osseous grafting.

\section{DISADVANTAGES OF NON-RESORBABLE MEMBRANES}

Membrane exposure caused by variable amounts of flap sloughing during healing has been a frequent post-surgical complication associated with the use of non-resorbable membranes [40]. Exposure rates as high as $31 \%$ resulting in GBR failure have been reported [59]. Membrane exposure permits a communication between the oral environment and newly forming tissues, increasing the potential for infection and decreasing the likelihood of regeneration. In addition, non-resorbable membranes must be retrieved by employing a second surgical procedure that can at times be a tedious undertaking and can also disturb healing [18].

\section{RESORBABLE MEMBRANES}

There are three types of biologically resorbable membranes: 1) polyglycoside synthetic copolymers: polylactic acid (Guidor ${ }^{\circledR}$ ), polyglactide $\left(\right.$ Resolute $^{\circledR}$ ), polyglactin $\left.910\left(\mathrm{Vicryl}^{\circledR}\right), 2\right)$ collagen and 3) calcium sulfate $\left(\right.$ CalcigenOral $\left.^{\circledR}\right)$.

\section{COLLAGEN MEMBRANES}

\section{Unique Properties of Collagen Membranes}

Collagen membranes share in common with all resorbable membranes the fact that they do not require a second surgery for retrieval. This saves time and cost and is 
also greatly appreciated by patients. Collagen is the principal component of connective tissue and provides structural support for tissues throughout the body [18].

Hemostasis: Collagen is hemostatic agent and possesses the ability to stimulate platelet attachment and to enhance fibrin linkage, which may facilitate initial clot formation and clot stabilization, leading to enhanced regeneration [18].

Chemotaxis: Collagen has been shown to be chemotactic for fibroblasts in vitro. This property could enhance cell migration in vivo [18].

Ease of manipulation: collagen can be easily manipulated and adapted [18].

Well tolerated: Collagen had been demonstrated to be a weak immunogen and is therefore well tolerated by patients [18]. Membranes made of bovine collagen do not elicit an antibody response when used in GTR.

Bioresorbable: Because collagen is bioresorbable, during enzymatic degradation it will incorporate with the flap to support new connective tissue attachment [60]. This may result in augmenting tissue/flap thickness to protect furtherbone formation.

Slow absorption: Membranes must remain in place until cells capable of regeneration are established at the wound site. Collagen membranes cross-linked with formaldehyde have been shown by Blumenthal [61] to last 6 to 8 weeks before being absorbed, whereas non-cross linked membranes lose their structural integrity in 7 days.

Degradation of resorbable membranes is accomplished by various mechanisms present within the periodontal tissues. The primary structural component of most commercially available collagen membranes is Type I collagen, which is degraded by endogenous collagenase into carbon dioxide and water. Cross-linkage of collagen fibers can affect the rate of degradation [62]. Cross-linking is a laboratory modification of the collagenous matrix designed to stabilize the collagen fibers and maintain the integrity of the membrane after placement [63]. There are many different laboratory techniques for cross-linking collagen membranes, including ultraviolet light and glutaraldehyde, which increases the time period that the device serves as an occlusive barrier in vivo.

In a report on the bio-degradation of various collagen membranes implanted in rats, Rothamel et al. [64] reported that non-cross linked porcine derived types I and III $\left(\right.$ BioGide $^{\mathbb{B}}$ ) exhibited a perfect tissue integration and thereby rapid vascularization, resulting in a nearly complete biodegradation 4 weeks following implantation without observable foreign body reactions. The cross-linked bovine collagen membrane (Ossix ${ }^{\mathrm{TM}}$ ) underwent the least amount of degradation after six months, maintaining greater thickness and occlusive function compared to porcine collagen (BioGide $^{\circledR}$ ), however it exhibited decreased tissue integration and vascularization and was associated with foreign body reactions. 3 other commercially available bovine collagen membranes $\left(\right.$ BioMend $^{\circledR}$, BioMendExtend ${ }^{\mathbb{R}}$, TutoDent ${ }^{\mathbb{B}}$ ), and three prototype collagen membranes VN (chemical crosslinked porcine type I and type III collagens) were included in the study. They concluded that the higher the degree of cross-linking, the longer the resorption rate.
Marinucci et al. [65] evaluated the in vitro influence of bioabsorbable and non-resorbable membranes on specific parameters of human osteoblast activity. Human osteoblasts were cultured on bioabsorbable membranes made of collagen, hyaluronic acid, and poly DL-lactide, and the most common non-resorbable membrane, which is made of ePTFE. The osteoblasts were cultured in vitro for 24 hours on barrier membranes in the presence of $3 \mathrm{H}$-thymidine and $3 \mathrm{H}-$ proline to study cell proliferation and collagen synthesis. Transforming growth factor-beta1 (TGF-beta1) secretion was evaluated in conditioned media using an ELISA kit. The results showed that collagen and poly DL-lactide stimulated DNA synthesis more than ePTFE and hyaluronic acid. All bioabsorbable membranes significantly increased collagen synthesis and alkaline phosphatase activity. Collagen and hyaluronic acid increased secretion of TGF- $\beta 1$, a growth factor involved in bone remodeling. These data suggest bioabsorbable membranes, particularly collagen and hyaluronic acid, may promote bone regeneration through their activity on osteoblasts.

Alpar et al. [66] evaluated the cytocompatibility of three resorbable and non-resorbable membranes in fibroblast and osteoblast-like cell cultures and observed the growth of those cells on the various barriers by scanning electron microscopy (SEM). Primary human periodontal ligament fibroblasts (HPLF) and human osteoblast-like cells (SAOS-2) were incubated with non-resorbable e-PTFE barriers and resorbable polylactic acid as well as collagen membranes. No changes were established in the periodontal ligament fibroblasts and human osteoblast-like cells after incubation with the collagen membrane. Cytotoxic effects, however, were induced by the polylactic acid barrier which slightly inhibited cell metabolism of the periodontal fibroblasts. Moderate cytotoxic reactions were caused by the e-PTFE membrane in HPLF-cultures and osteoblast-like cell monolayers. Mitochondrial activity in both cell cultures was significantly reduced by e-PTFE barriers in comparison to non-incubated control cells. SEM analysis of cell behavior on barriers demonstrated the differences between these materials: collagen barriers were densely populated with HPLF and SAOS-2, whereas only few or no cells were seen to adhere to the ePTFE and polylactic acid membranes. The collagen barrier investigated is very cytocompatible and may be integrated into connective tissue well. On the contrary, the ePTFE and polylactic acid membranes induced slight to moderate cytotoxic reactions, which may reduce cellular adhesion. Thus, gap formation between the barrier surface and the connective tissue may be promoted which may facilitate epithelial down-growth and microbial accumulation. Consequently, these effects may reduce the potential gain in periodontal attachment.

Collagen Membrane in GBR-Ridge Augmentation GBR procedures utilizing collagen membranes have been widely reported.

Sevor et al. [67] evaluated the usefulness of resorbable collagen membrane for guided tissue regeneration. Buccal dehiscences were surgically induced in 6 dog mandibles.

Twenty-four hydroxyapatite-coated and twenty-four gritblasted implants were then placed in a random pattern in both sides of the mandibles (two of each type of implant in each side of the mandible). A resorbable collagen barrier 
membrane was placed around one pair of implants on each side. The other two implants on each side served as controls. At 8 weeks, the mean defect fill was $80.29 \%$ in the collagen membrane-treated group compared to $38.62 \%$ in the control group. Sites around experimental hydroxyapatite-coated and grit-blasted implants showed significantly more bone fill than did control sites. In addition to its apparent ability to encourage bone regeneration, the collagen membrane is resorbable, obviating the need for a second surgery to allow removal.

In a pilot study [68], Colangelo et al. created through and through defects on the lateral aspect of rabbit mandibles and then treated these defects with either a type I highly crosslinked collagen membrane or no-treatment control. The histologic evaluation at 30 days demonstrated a nearly complete continuous layer of lamellar bone with osteoblastic activity in the collagen membrane-treated group compared to only fibrous connective tissue in the control group.

In another study, Zitzmann et al. [69] compared a resorbable collagen membrane $\left(\right.$ Bio-Gide ${ }^{\circledR}$ ) to the conventional e-PTFE material (Gore-Tex ${ }^{B}$ ) for guided bone regeneration in situations involving exposed implant surfaces. Over a 2-year period, 25 split-mouth patients were treated randomly: one defect site was treated with Bio-Gide ${ }^{\circledR}$ and the other defect site with Gore-Tex ${ }^{\circledR}$; all 84 defects were filled with Bio-Oss ${ }^{\circledR}$ and covered with the respective membrane. Changes in defect surface for both types of membranes were statistically significant. The mean average percentage of bone fill was $92 \%$ for Bio-Gide ${ }^{\circledR}$ and $78 \%$ for Gore-Tex ${ }^{\circledR}$ sites. In the latter group, $44 \%$ wound dehiscences and/or premature membrane removal occurred. The resorbable membrane, Bio-Gide, in combination with a bone graft, can be a useful alternative to the well-established ePTFE membranes.

Recently, Parodi et al. [70] evaluated the possibility of expanding an edentulous ridge spanning two or more teeth by a two-step technique with bio-resorbable collagen membranes. Sixteen healthy patients were treated. The borderline of the crest width was less than or equal to $4 \mathrm{~mm}$. The width of the crest at the location of the surgical stent was measured at the time of GBR procedure and 7 to 12 months later during implant insertion. Native collagen sponges were placed buccally and lingually, and a collagen membrane was shaped and trimmed to completely cover the edentulous ridge. The patients were recalled every 2 weeks. At implant placement, the mean increase in the size of the crest was $2.49 \mathrm{~mm}$. In 12 out of 16 patients $(75 \%)$ it was possible to insert 27 implants according to the prosthetic need established previously. All implants were successfully loaded, and in the four cases where no appreciable results were obtained, no clinical complications or loss of hard and soft tissue were observed.

Another study [71] aimed to clinically and histomorphometrically compare two collagen membranes, Bio-Gide $^{\circledR}$ and BioMend ExtendTM, for the treatment of implant dehiscence defects in eight mongrel dogs. Implant dehiscence defects were surgically created in edentulous ridges, followed by the placement of three endosseous implants bilaterally in the mandible. Each implant dehiscence defect was randomly assigned to one of three treatment groups: (1) control (no membrane), (2) porcine dermis collagen barrier (Bio-Gide ${ }^{\circledR}$ ) or (3) bovine tendon collagen barrier (BioMend ExtendTM). Dogs were sacrificed at 4 and 16 weeks (four dogs each) after treatment. The results of the study revealed no significant differences among groups for any parameter at 4 weeks. However, at 16 weeks, more linear bone fill (LF), bone-to- implant-contact (BIC), and new bone fill were noted in the membrane-treated groups than controls. BioMend Extend-treated defects demonstrated significantly greater BIC than control at this time point. BIC at 16 weeks was significantly greater than 4week BIC. Membrane exposure occurred in 9 out of 15 sites examined, resulting in significantly less LF and BIC than the sites without membrane exposure. The results of this study indicate that:

(1) GBR treatment with collagen membranes may significantly enhance bone regeneration, manifested at late stage (16 weeks) of healing; and (2) space maintenance and membrane coverage were the two most important factors affecting GBR using bioabsorbable collagen membranes.

Taguchi et al. [72] focused on histological changes and cellular events in osteogenesis induced by GBR with a collagenous membrane, Bio-Gide ${ }^{\circledR}$. This membrane appears to have osteoconductivity, resulting in a well-augmented alveolar ridge, which is suitable for the subsequent placement of dental implants. This material represents an excellent choice for GBR by promoting osteoblastic activity.

\section{GBR WITH BONE GRAFTING}

Various methods have been developed to prevent membrane collapse and to preserve and maintain the space. Placing various bone graft materials under the membrane or using mechanical support are among the methods used. Grafts are generally classified according to their original source as follows: autograft (oral or extraoral), allograft (DFDBA, FDBA, Puros cancellous), xenograft (bovine or porcine), and alloplasts (hydroxyapatite, calcium phosphate).

\section{Autogenous Bone Grafts}

Iliac bone and marrow autografts have proven to be the most predictable graft materials for bone growth. However, because of the necessity of harvesting from a secondary surgical site and the possible morbidity associated with these procedures, they are no longer popular1. Complications associated with the use of fresh iliac bone and marrow included a high rate of root resorption and ankylosis [73]. These complications were later minimized by either freezing the bone graft in a storage medium or adding autologous intraoral bone to the harvested iliac crest bone graft mixture.

Intraoral autogenous bone grafts have been harvested from various intraoral sites including edentulous ridges, the maxillary tuberosity, 8- to 12 -week post-extraction healing sites, and tori or exostoses1. One controlled study [74] of 37 paired defects demonstrated $2.98 \mathrm{~mm}$ of bone gain when autogenous intraoral bone grafts were used, as compared with $0.66 \mathrm{~mm}$ for debrided controls that received no grafts.

The source of intraoral bone also is important [1]. When bone is predominantly cortical in nature, it has little osteogenic potential. Cancellous bone, which contains hematopoietic marrow, such as red bone marrow from the 
maxillary tuberosity or from healing bone sockets 8 to 12 weeks after extraction, provides better osteogenic potentiall.

\section{GBR WITH BONE GRAFTS}

Proussaefs [75] reported on three patients who underwent localized alveolar ridge augmentation using block autografts harvested from the mandibular tori. Autogenous particulate bone graft was placed at the periphery of the block. Resorbable collagen membrane was placed above the graft material. Implant placement surgery followed at 6 to 16 months after bone grafting. Clinical evaluation revealed incorporation of the graft material at the recipient site. Histologic evaluation suggested that the block autograft was vital and in an active remodeling phase at the time of implant placement. Impressions were made intraorally before and 6 months after bone grafting. Laboratory measurements revealed $13 \%$ resorption at 6 months after bone grafting while $0.53 \mathrm{~mL}$ of ridge augmentation was achieved 6 months after bone grafting. Linear tomographs indicated $4.33 \mathrm{~mm}$ of lateral alveolar ridge augmentation. This report suggests that block autografts harvested from the mandibular tori may have the potential to maintain their vitality after bone grafting, while they may demonstrate resorption rates similar to those of autografts harvested from other intraoral donor sites.

Llambes et al. [76] performed vertical ridge augmentation on 11 patients at the time of implant placement. The part of the implant out of bone was covered with autogenous bone/graft, and a slow-resorption collagen membrane was placed on top.

Second-stage surgery was performed 4 to 6 months later, and healing abutments were placed. Measurements revealed that the mean implant out of bone was $3.5 \mathrm{~mm}$ at stage 1 and $0.5 \mathrm{~mm}$ at stage 2. Mean bone gain was $3 \mathrm{~mm}$, which represented $83 \%$ of the exposed implant at stage 1. Minimal complications were detected, and only one case failed. Histology from one successful case showed new trabecular bone with large cellular marrow spaces in the regenerated area. Slow-resorption collagen membranes have the potential to promote vertical ridge augmentation when used with autogenous bone at the time of implant placement.

The clinical outcome of horizontal ridge augmentation using autogenous block grafts covered with an organic bovine bone mineral (ABBM) and a bioabsorbable collagen membrane was analysed by von Arx and Buser [77]. In 42 patients with severe horizontal bone atrophy, a staged approach was chosen for implant placement following horizontal ridge augmentation. A block graft was harvested from the symphysis or retromolar area, and secured to the recipient site with fixation screws. The block graft was subsequently covered with ABBM and a collagen membrane. Following a mean healing period of 5.8 months, the fifty-eight augmented sites were re-entered, and the crest width was re-assessed prior to implant placement. The mean initial crest width measured $3.06 \mathrm{~mm}$. At re-entry, the mean width of the ridge was $7.66 \mathrm{~mm}$, with a calculated mean gain of horizontal bone thickness of $4.6 \mathrm{~mm}$. Only minor surface resorption of $0.36 \mathrm{~mm}$ was observed from augmentation to re-entry. The presented technique of ridge augmentation using autogenous block grafts with ABBM filler and collagen membrane coverage demonstrated successful horizontal ridge augmentation with high predictability. The hydrophilic membrane was easy to apply, and did not cause wound infection in the rare instance of membrane exposure.

In a recent study, Hammerle et al. [78] tested whether or not resorbable membranes and bone substitutes will lead to successful horizontal ridge augmentation allowing implant installation under standard conditions. Twelve patients in need of implant therapy participated in this study. Soft tissue flaps were carefully raised and blocks or particles of deproteinized bovine bone mineral (DBBM/ Bio-Oss ${ }^{\circledR}$ ) were placed in the defect area. A collagenous membrane (BioGide $^{(B)}$ ) was applied to cover the DBBM and was fixed to the surrounding bone using poly-lactic acid pins. No flap dehiscences and no exposures of membranes were observed. Nine to 10 months following augmentation surgery, flaps were raised in order to visualize the outcomes of the augmentation. An integration of the DBBM particles into the newly formed bone was consistently observed. Merely on the surface of the new bone, some pieces of the grafting material were only partly integrated into bone. However, these were not encapsulated by connective tissue but rather anchored into the newly regenerated bone. In all of the cases but one, the bone volume following regeneration was adequate to place implants in a prosthetically ideal position. Before the regenerative procedure, the average crestal bone width was $3.2 \mathrm{~mm}$ and increased to $6.9 \mathrm{~mm}$ at the time of implant placement. This difference was statistically significant. After a healing period of 9-10 months, the combination of DBBM and a collagen membrane is an effective treatment option for horizontal bone augmentation before implant placement.

\section{PERICARDIUM MEMBRANES}

Bovine pericardium has been widely used for grafts in cardiac surgery and seems to have suitable properties for use as a dural graft. Filippi et al. [79] reported on the use of solvent-preserved, gamma-sterilized Tutoplast ${ }^{\circledR}$ bovine pericardium for dural grafts in 32 patients undergoing cranial and spinal operations with the objective of clinically assessing this material and technique by a retrospective analysis.

Outcomes were excellent in 31 patients; the one poor outcome was unrelated to surgical closure. Bovine pericardium was found to be a flexible and easily suturable, safe and cost-effective material for duraplasty.

Also Bovine pericardium has been used in abdominal wall repair (incisional hernias). In an experimental study by Kapan et al. [80], three commonly used mesh materials (Gore-Tex $^{\circledR}$ PTFE; Tutoplast ${ }^{\circledR}$ Fascia lata; Tutopatch ${ }^{\circledR}$ Pericardium bovine) were compared according to effectiveness, strength, adhesion formation, histological changes, and early complications. Three groups, each consisting of 14 rats, have been formed as group A: polytetrafluoroethylene (PTFE), group B: pericardium bovine and group $\mathrm{C}$ : fascia lata. No statistically significant difference regarding adhesion formation was observed between groups although adhesion formation was less significant in PTFE and pericardium bovine groups than in the fascia lata group. No statistically significant difference was observed between groups regarding wound maturation. In this experimental model, PTFE and pericardium bovine 
were found to be superior to fascia lata in abdominal wall repair.

Bovine pericardium was also tested as a collagen membrane for exclusion of all non-osteogenic cells from the healing wound site, and for protection and stabilization of the healing clot. In a rat study by Schwarz et al. [81], in histologic and histometric analysis after 8 weeks following implantation, the pericardium membrane showed approximately $60 \%$ of membrane thickness measured after 2 weeks. The Tutodent ${ }^{\circledR}$ membrane body seemed to be structured like an interconnected porous system. Histologic analysis 2 weeks following implantation revealed that merely half the Tutodent ${ }^{\circledR}$ membrane body was vascularized. After 16 weeks, the Tutodent ${ }^{\circledR}$ was almost entirely organized and replaced by newly formed connective tissue. After 24 weeks, a nearly complete biodegradation and substitution of the membrane by newly formed connective tissue was observed. They also described the resorption time at 8-16 weeks. Another study from Rothamel et al. [82] showed that the pericardium membrane promoted the attachment and proliferation of human periodontal ligament fibroblasts and human osteoblasts. Steigmann [83] evaluated the clinical feasibility of using a native collagen physical resorbable barrier made of bovine pericardium to augment localized alveolar ridge defects for the subsequent placement of dental implants. There were 8 systemically healthy patients with 19 implant sites, with inadequate dental alveolar ridge widths, selected for study. All ridge defects were augmented with bovine xenograft and a collagen pericardium membrane. Horizontal (width) hard tissue measurements were taken the day of ridge augmentation surgery, or implant placement and augmentation (baseline), and at the 6-month (reentry or uncovering) surgery. The change in ridge width varied from a loss of $0.2 \mathrm{~mm}$ to a gain of $7.8 \mathrm{~mm}$, measured clinically with a mean value of 3.04 and a median of $2.8 \mathrm{~mm}$ from baseline.

The results suggested that pericardium collagen membrane might be a suitable component for augmentation of localized alveolar ridge defects in conjunction with different xenografts.

Puros $^{\circledR}$ Pericardium is a natural biological dressing designed for guided tissue regeneration and guided bone regeneration procedures. It retains the natural collagen matrix and mechanical properties of native pericardium due to the proprietary Tutoplast ${ }^{\circledR}$ Process. It is characterized by its multidirectional strength, quick hydration, five-year shelf life and room temperature storage. Puros ${ }^{\circledR}$ Pericardium has a resorption profile of 4 to 6 months. The timeframe for remodeling depends upon the site; patient age, health and metabolic and nutritional status; biomechanical load on the graft. Three convenient sizes can be cut to shape for specific procedures.

The Puros ${ }^{\circledR}$ Allograft family of products is refined using the proprietary, 5stage Tutoplast ${ }^{\mathbb{B}}$ sterilization process. While preserving the valuable collagen matrix and tissue integrity, this process gently removes unwanted materials such as cells, antigens and viruses, as well as inactivating pathogens. Osmotic treatment ruptures the cell membranes killing bacteria, washes out cellular debris and removes antigens.
Oxidative treatment destroys soluble proteins, nonenveloped viruses and bacterial spores and minimizes potential graft rejection. Alkaline treatment reduces prion infectivity. Solvent dehydration removes any residual prions, inactivates enveloped viruses, and dehydrates the tissue, allowing it to be stored at room temperature.

Limited-dose gamma irradiation provides a sterility level of 10-6 and preserves graft integrity.

Shin and Sohn [84] described a technique regarding repairing completely perforated sinus membrane after the removal of a mucocele using human collagen pericardium membrane (Tutoplast ${ }^{\circledR}$ pericardium) and fibrin adhesive $\left(\right.$ Greenplast $\left.^{(}\right)$to stabilize collagen membrane.

In a clinical report, Taskonak and Ozkan [85] described guided bone augmentation for treatment of a facial maxillary alveolar bone defect to enhance the esthetic result for an allceramic fixed partial denture (FPD). A combination of decalcified freeze-dried bone allograft and resorbable human pericardium, in conjunction with cortical channel expansion, was used for the augmentation process to eliminate a secondary surgical procedure. Post-operative examinations showed improvement in the alveolar bone contour. The regeneration of the missing osseous structure was accomplished to support the future esthetic soft tissue contours. This osseous regenerative technique significantly increased the functional and esthetic outcome of the final FPD by restoring the alveolar ridge defect to its original dimension.

Bovine and human pericardium membranes were also evaluated in a study by Thomaidis [86]. Fifty adult male New Zealand white rabbits were used in this study.

Five groups of 10 animals each were used: HFL (human fascia lata membrane), HP (human pericardium), HFT (human fascia temporalis), BP (bovine pericardium), and ePTFE (expanded polytetrafluoroethylene). Animals were sacrificed 10 weeks after membrane application. In each animal, 9-mm circular mandibular defects were created bilaterally. On 1 side of the jaw, the defect was covered with 1 of the test membranes; the defect on the other side served as a control. Membranes were significantly superior to the controls in all animals. Paired comparisons showed that groups HFL, HP, BP, and e-PTFE were significantly superior to HFT. Conversely, comparisons of HFL-HP, HFL-BP, HFL-PTFE, HP-BP, HP-ePTFE, and BP-ePTFE, showed no significant differences. According to the results, the fascia lata, human pericardium, bovine pericardium, and e-PTFE advance bone regeneration and can be successfully used as GBR membranes for osseous defects beyond the critical size.

The defect in the human pericardium group was filled with a mature, mainly newly formed bone, lamellar in its greatest part, with distinct bone trabeculae and marrow sites. The thickness of the new bone was slightly smaller than normal bone.

The gap was fully bridged by new bone, whose bone trabeculae were almost sufficiently thick around the bone tissue. The membrane was visible without causing any inflammatory reaction. In group BP, the results were the same as those in group HP. The origin of the pericardium 
(human or bovine) did not seem to influence the process of bone regeneration.

\section{REFERENCES}

[1] Rose L, Mealey B, Genco R, Cohen W. Periodontics: medicine, surgery, and implants: Elsevier: Mosby 2004.

[2] Melcher AH. On the repair potential of periodontal tissues. J Periodontol 1976; 47: 256-60.

[3] Aukhil I, Simpson DM, Suggs C, Pettersson E. In vivo differentiation of progenitor cells of the periodontal ligament. An experimental study using physical barriers. J Clin Periodontol 1986; 13: 862-8.

[4] Isidor F, Karring T, Nyman S, Lindhe J. The significance of coronal growth of periodontal ligament tissue for new attachment formation. $\mathrm{J}$ Clin Periodontol 1986; 13: 145-50.

[5] Iglhaut J, Aukhil I, Simpson DM, Johnston MC, Koch G. Progenitor cell kinetics during guided tissue regeneration in experimental periodontal wounds. J Periodontal Res 1988; 23: 107-17.

[6] Melcher AH, McCulloch CA, Cheong T, Nemeth E, Shiga A. Cells from bone synthesize cementum-like and bone-like tissue in vitro and may migrate into periodontal ligament in vivo. J Periodontal Res 1987; 22: 246-7.

[7] Nyman S, Lindhe J, Karring T, Rylander H. New attachment following surgical treatment of human periodontal disease. J Clin Periodontol 1982; 9: 290-6.

[8] Gottlow J, Nyman S, Lindhe J, Karring T, Wennstrom J. New attachment formation in the human periodontium by guided tissue regeneration. Case reports. J Clin Periodontol 1986; 13: 604-16.

[9] Nyman S, Gottlow J, Lindhe J, Karring T, Wennstrom J. New attachment formation by guided tissue regeneration. J Periodontal Res 1987; 22: 252-4.

[10] Stavropoulos F, Nale JC, Ruskin JD. Guided bone regeneration. Oral Maxillofac Surg Clin North Am 2002; 14: 15-27.

[11] Dahlin C, Gottlow J, Lindhe A, Nyman S. Healing of maxillary and mandibular bone defects using a membrane technique. An experimental study in monkeys. Scand J Plast Reconstr Surg Hand Surg 1990; 24: 13-9.

[12] Dahlin C, Linde A, Gottlow J, Nyman S. Healing of bone defects by guided tissue regeneration. Plast Reconstr Surg 1988 ; 81: 672-6.

[13] Dahlin C, Sennerby L, Lekholm U, Linde A, Nyman S. Generation of new bone around titanium implants using a membrane technique: an experimental study in rabbits. Int J Oral Maxillofac Implants 1989; 4: 19-25.

[14] Urist MR, McLean FC. Recent advances in physiology of bone. I. J Bone Joint Surg Am 1963; 45: 1305-13.

[15] Schropp L, Wenzel A, Kostopoulos L, Karring T. Bone healing and soft tissue contour changes following single-tooth extraction: a clinical and radiographic 12-month prospective study. Int J Periodontics Restorative Dent 2003; 23: 313-23.

[16] Iasella JM, Greenwell $\mathrm{H}$, Miller RL, et al. Ridge preservation with freeze-dried bone allograft and a collagen membrane compared to extraction alone for implant site development: a clinical and histologic study in humans. J Periodontol 2003; 74: 990-9.

[17] Buser D, Dula K, Belser U, Hirt HP, Berthold H. Localized ridge augmentation using guided bone regeneration. 1. Surgical procedure in the maxilla. Int J Periodontics Restorative Dent 1993; 13: 29-45.

[18] Wang HL, Carroll MJ. Guided bone regeneration using bone grafts and collagen membranes. Quintessence Int 2001; 32: 504-15.

[19] Kornman KS, Robertson PB. Fundamental principles affecting the outcomes of therapy for osseous lesions. Periodontology 2000; 22: 2243.

[20] Passariello C, Thaller MC, Selan L, Berlutti F, de Luca M, Renzini G. Periodontal regeneration procedures may induce colonization by glycocalyxproducing bacteria. Med Microbiol Immunol 1991; 180: 6772.

[21] Mombelli A, Lang NP, Nyman S. Isolation of periodontal species after guided tissue regeneration. J Periodontol 1993; 64: 1171-5.

[22] Selvig KA, Nilveus RE, Fitzmorris L, Kersten B, Khorsandi SS. Scanning electron microscopic observations of cell populations and bacterial contamination of membranes used for guided periodontal tissue regeneration in humans. J Periodontol 1990; 61: 515-20.

[23] Simion M, Trisi P, Maglione M, Piattelli A. A preliminary report on a method for studying the permeability of expanded polytetrafluoroethylene membrane to bacteria in vitro: a scanning electron microscopic and histological study. J Periodontol 1994; 65: 755-61.
[24] Mombelli A, Zappa U, Bragger U, Lang NP. Systemic antimicrobial treatment and guided tissue regeneration. Clinical and microbiological effects in furcation defects. J Clin Periodontol 1996; 23: 386-96.

[25] Nowzari H, London R, Slots J. The importance of periodontal pathogens in guided periodontal tissue regeneration and guided bone regeneration. Compend Contin Educ Dent 1995; 16: 1042-6.

[26] Wang HL, Yuan K, Burgett F, Shyr Y, Syed S. Adherence of oral microorganisms to guided tissue membranes: an in vitro study. J Periodontol 1994; 65: 211-8.

[27] Sander L, Karring T. New attachment and bone formation in periodontal defects following treatment of submerged roots with guided tissue regeneration. J Clin Periodontol 1995; 22: 295-9.

[28] Cortellini P, Pini-Prato G, Tonetti M. Periodontal regeneration of human infrabony defects (V). Effect of oral hygiene on long-term stability. J Clin Periodontol 1994; 21: 606-10.

[29] Machtei EE, Cho MI, Dunford R, Norderyd J, Zambon JJ, Genco RJ. Clinical, microbiological, and histological factors which influence the success of regenerative periodontal therapy. J Periodontol 1994; 65: 154-61

[30] Falk H, Laurell L, Ravald N, Teiwik A, Persson R. Guided tissue regeneration therapy of 203 consecutively treated intrabony defects using a bioabsorbable matrix barrier. 571581. Clinical and radiographic findings. J Periodontol 1997; 68: 571-81.

[31] Hugoson A, Ravald N, Fornell J, Johard G, Teiwik A, Gottlow J. Treatment of class II furcation involvements in humans with bioresorbable and non-resorbable guided tissue regeneration barriers. A randomized multi-center study. J Periodontol 1995; 66: 624-34.

[32] Rosenberg ES, Cutler SA. The effect of cigarette smoking on the longterm success of guided tissue regeneration: a preliminary study. Ann R Australas Coll Dent Surg 1994; 12: 89-93.

[33] Tonetti MS, Pini-Prato G, Cortellini P. Effect of cigarette smoking on periodontal healing following GTR in infrabony defects. A preliminary retrospective study. J Clin Periodontol 1995; 22: 229-34.

[34] Cortellini P, Paolo G, Prato P, Tonetti MS. Long-term stability of clinical attachment following guided tissue regeneration and conventional therapy. J Clin Periodontol 1996; 23: 106-11.

[35] Schwartz-Arad D, Levin L, Sigal L. Surgical success of intraoral autogenous block onlay bone grafting for alveolar ridge augmentation. Implant Dent 2005; 14: 131-8.

[36] Tonetti MS, Pini-Prato G, Cortellini P. Periodontal regeneration of human intrabony defects. IV. Determinants of healing response. J Periodontol 1993; 64: 934-40.

[37] Tonetti MS, Prato GP, Cortellini P. Factors affecting the healing response of intrabony defects following guided tissue regeneration and access flap surgery. J Clin Periodontol 1996; 23: 548-56.

[38] Kaldahl WB, Kalkwarf KL, Patil KD, Molvar MP, Dyer JK. Long-term evaluation of periodontal therapy: II. Incidence of sites breaking down J Periodontol 1996; 67: 103-8.

[39] Reynolds MA, Bowers GM. Periodontal regeneration following surgical treatment. Curr Opin Periodontol 1996; 3: 126-39.

[40] Murphy KG. Postoperative healing complications associated with Gore-Tex periodontal material. Part I. Incidence and characterization. Int J Periodontics Restorative Dent 1995; 15: 363-75.

[41] Machtei EE. The effect of membrane exposure on the outcome of regenerative procedures in humans: a meta-analysis. J Periodontol 2001; 72: 512-6.

[42] Simion M, Baldoni M, Zaffe D. Guided tissue regeneration in osseointegrated implants. II: extraction sockets. Ital J Osseointegration 1991; 1: 40-5.

[43] Becker W, Dahlin C, Becker BE, et al. The use of e-PTFE barrier membranes for bone promotion around titanium implants placed into extraction sockets: a prospective multicenter study. Int $\mathrm{J}$ Oral Maxillofac Implants 1994; 9: 31-40.

[44] Simion M, Trisi P, Piattelli A. Vertical ridge augmentation using a membrane technique associated with osseointegrated implants. Int J Periodontics Restorative Dent 1994; 14: 496-511.

[45] Mellonig JT, Triplett RG. Guided tissue regeneration and endosseous dental implants. Int J Periodontics Restorative Dent 1993; 13: 108-19.

[46] Shanaman RH. A retrospective study of 237 sites treated consecutively with guided tissue regeneration. Int J Periodontics Restorative Dent 1994; 14: 292-301.

[47] Anderegg CR, Metzler DG, Nicoll BK. Gingiva thickness in guided tissue regeneration and associated recession at facial furcation defects. J Periodontol 1995; 66: 397-402.

[48] Kramer GM. Surgical alternatives in regenerative therapy of the periodontium. Int J Periodontics Restorative Dent 1992; 12: 10-31. 
[49] Jovanovic SA, Nevins M. Bone formation utilizing titanium-reinforced barrier membranes. Int J Periodontics Restorative Dent 1995; 15: 56-69.

[50] Greenstein G, Caton JG. Biodegradable barriers and guided tissue regeneration. Periodontology 2000 1993; 1: 36-45.

[51] Hardwick R, Hayes BK, Flynn C. Devices for dentoalveolar regeneration: an up-to-date literature review. J Periodontol 1995; 66: 495-505.

[52] Scantlebury TV. 1982-1992: a decade of technology development for guided tissue regeneration. J Periodontol 1993; 64: 1129-37.

[53] Buser D, Bragger U, Lang NP, Nyman S. Regeneration and enlargement of jaw bone using guided tissue regeneration. Clin Oral Implants Res 1990; 1:22-32.

[54] Schenk RK, Buser D, Hardwick WR, Dahlin C. Healing pattern of bone regeneration in membrane-protected defects: a histologic study in the canine mandible. Int J Oral Maxillofac Implants 1994; 9: 13-29.

[55] Buser D, Ruskin J, Higginbottom F, Hardwick R, Dahlin C, Schenk RK. Osseointegration of titanium implants in bone regenerated in membrane- protected defects: a histologic study in the canine mandible. Int J Oral Maxillofac Implants 1995; 10: 666-81.

[56] Fiorellini JP, Kim DM, Nakajima Y, Weber HP. Osseointegration of titanium implants following guided bone regeneration using expanded polytetrafluoroethylene membrane and various bone fillers. Int $\mathrm{J}$ Periodontics Restorative Dent 2007; 27: 287-94.

[57] Simion M, Dahlin C, Trisi P, Piattelli A. Qualitative and quantitative comparative study on different filling materials used in bone tissue regeneration: a controlled clinical study. Int J Periodontics Restorative Dent 1994; 14: 15-198.

[58] Nevins M, Mellonig JT. Enhancement of the damaged edentulous ridge to receive dental implants: a combination of allograft and the GORETEX membrane. Int J Periodontics Restorative Dent 1992; 12: 96-111.

[59] Lang NP, Hammerle CH, Bragger U, Lehmann B, Nyman SR. Guided tissue regeneration in jawbone defects prior to implant placement. Clin Oral Implants Res 1994; 5: 7-92.

[60] Pitaru S, Tal H, Soldinger M, Noff M. Collagen membranes prevent apical migration of epithelium and support new connective tissue attachment during periodontal wound healing in dogs. J Periodontal Res 1989; 24: 247-53.

[61] Blumenthal NM. The use of collagen membranes to guide regeneration of new connective tissue attachment in dogs. J Periodontol 1988; 59: 830-6.

[62] Minabe M, Kodama T, Kogou T, et al. Different cross-linked types of collagen implanted in rat palatal gingiva. J Periodontol 1989; 60: 35-43.

[63] Charulatha V, Rajaram A. Influence of different crosslinking treatments on the physical properties of collagen membranes. Biomaterials 2003; 24: 759-67.

[64] Rothamel D, Schwarz F, Sager M, Herten M, Sculean A, Becker J. Biodegradation of differently cross-linked collagen membranes: an experimental study in the rat. Clin Oral Implants Res 2005; 16: 369-78.

[65] Marinucci L, Lilli C, Baroni T, et al. In vitro comparison of bioabsorbable and non-resorbable membranes in bone regeneration. $\mathrm{J}$ Periodontol 2001; 72: 753-9.

[66] Alpar B, Leyhausen G, Gunay H, Geurtsen W. Compatibility of resorbable and non-resorbable guided tissue regeneration membranes in cultures of primary human periodontal ligament fibroblasts and human osteoblast-like cells. Clin Oral Investig 2000; 4: 219-25.

[67] Sevor JJ, Meffert RM, Cassingham RJ. Regeneration of dehisced alveolar bone adjacent to endosseous dental implants utilizing a resorbable collagen membrane: clinical and histologic results. Int J Periodontics Restorative Dent 1993; 13: 71-83.
[68] Colangelo P, Piattelli A, Barrucci S, Trisi P, Formisano G, Caiazza S. Bone regeneration guided by resorbable collagen membranes in rabbits: a pilot study. Implant Dent 1993; 2: 101-5.

[69] Zitzmann NU, Naef R, Scharer P. Resorbable versus nonresorbable membranes in combination with Bio-Oss for guided bone regeneration. Int J Oral Maxillofac Implants 1997; 12: 844-52.

[70] Parodi R, Carusi G, Santarelli G, Nanni F. Implant placement in large edentulous ridges expanded by GBR using a bioresorbable collagen membrane. Int J Periodontics Restorative Dent 1998; 18: 266-75.

[71] Oh TJ, Meraw SJ, Lee EJ, Giannobile WV, Wang HL. Comparative analysis of collagen membranes for the treatment of implant dehiscence defects. Clin Oral Implants Res 2003; 14: 80-90.

[72] Taguchi Y, Amizuka N, Nakadate M, et al. A histological evaluation for guided bone regeneration induced by a collagenous membrane. Biomaterials 2005; 26: 6158-66.

[73] Schallhorn RG. Postoperative problems associated with iliac transplants. J Periodontol 1972; 43: 3-9.

[74] Froum SJ, Ortiz M, Witkin RT, Thaler R, Scopp IW, Stahl SS. Osseous autografts. III. Comparison of osseous coagulum-bone blend implants with open curettage. J Periodontol 1976; 47: 287-94.

[75] Proussaefs P. Clinical and histologic evaluation of the use of mandibular tori as donor site for mandibular block autografts: report of three cases. Int J Periodontics Restorative Dent 2006; 26: 43-51.

[76] Llambes F, Silvestre FJ, Caffesse R. Vertical guided bone regeneration with bioabsorbable barriers. J Periodontol 2007; 78: 2036-42.

[77] von Arx T, Buser D. Horizontal ridge augmentation using autogenous block grafts and the guided bone regeneration technique with collagen membranes: a clinical study with 42 patients. Clin Oral Implants Res 2006; 17: 359-66.

[78] Hammerle CH, Jung RE, Yaman D, Lang NP. Ridge augmentation by applying bioresorbable membranes and deproteinized bovine bone mineral: a report of twelve consecutive cases. Clin Oral Implants Res 2008; 19: 19-25.

[79] Filippi R, Schwarz M, Voth D, Reisch R, Grunert P, Perneczky A. Bovine pericardium for duraplasty: clinical results in 32 patients. Neurosurg Rev 2001; 24: 103-7.

[80] Kapan S, Kapan M, Goksoy E, Karabicak I, Oktar H. Comparison of PTFE, pericardium bovine and fascia lata for repair of incisional hernia in rat model, experimental study. Hernia 2003; 7: 39-43.

[81] Schwarz F, Rothamel D, Herten M, Sager M, Becker J. Angiogenesis pattern of native and cross-linked collagen membranes: an immunohistochemical study in the rat. Clin Oral Implants Res 2006; 17: 403-9.

[82] Rothamel D, Schwarz F, Sculean A, Herten M, Scherbaum W, Becker $\mathrm{J}$. Biocompatibility of various collagen membranes in cultures of human PDL fibroblasts and human osteoblast-like cells. Clin Oral Implants Res 2004; 15: 443-9.

[83] Steigmann M. Pericardium membrane and xenograft particulate grafting materials for horizontal alveolar ridge defects. Implant Dent 2006; 15: 186-91.

[84] Shin HI, Sohn DS. A method of sealing perforated sinus membrane and histologic finding of bone substitutes: a case report. Implant Dent 2005; 14: 328-33.

[85] Taskonak B, Ozkan Y. An alveolar bone augmentation technique to improve esthetics in anterior ceramic FPDs: a clinical report. J Prosthodont 2006; 15: 32-6.

[86] Thomaidis V, Kazakos K, Lyras DN, et al. Comparative study of 5 different membranes for guided bone regeneration of rabbit mandibular defects beyond critical size. Med Sci Monit 2008; 14: 67-73. 